RUNNING HEAD: LYNX M2 AND IRREVERSIBILE EVOLUTION

\title{
Return of a lost structure in the evolution of felid dentition revisited: A DevoEvo perspective on the irreversibility of evolution
}

\author{
Vincent J. Lynch
}

Department of Biological Sciences, University at Buffalo, SUNY, 551 Cooke Hall, Buffalo, NY, 14260, USA. Correspondence: vjlynch@buffalo.edu 
RUNNING HEAD: LYNX M2 AND IRREVERSIBILE EVOLUTION

\section{Abstract}

There is a longstanding interest in whether the loss of complex characters is reversible (so-called "Dollo's law"). Reevolution has been suggested for numerous traits but among the first was Kurtén (1963), who proposed that the presence of the second lower molar $\left(\mathrm{M}_{2}\right)$ of the Eurasian lynx (Lynx lynx) was a violation of Dollo's law because all other Felids lack $\mathrm{M}_{2}$. While an early and often cited example for the reevolution of a complex trait, Kurtén (1963) and Werdelin (1987) used an ad hoc parsimony argument to support their proposition that $\mathrm{M}_{2}$ reevolved in Eurasian lynx. Here I revisit the evidence that $\mathrm{M}_{2}$ reevolved in Eurasian lynx using explicit parsimony and maximum likelihood models of character evolution and find strong evidence that Kurtén (1963) and Werdelin (1987) were correct - $M_{2}$ reevolved in Eurasian lynx. Next, I explore the developmental mechanisms which may explain this violation of Dollo's law and suggest that the reevolution of lost complex traits may arise from the reevolution of cisregulatory elements and protein-protein interactions, which have a longer half-life after silencing that protein coding genes. Finally, I present a model developmental model to explain the reevolution $M_{2}$ in Eurasian lynx.

\section{Introduction}

There is a growing interest and renewed debate in whether the loss of complex characters is reversible. Reevolution of complex characters has been suggested for eyes in ostracods (Dingle, 2003; Hunt, 2007; Oakley, 2003), spiders (West-Ebehard, 2003), and snakes (Coates and Rutta, 2000; Laurent, 1983), ocelli in cave crickets (Desutter-Grandcolas, 1993), wings in water spiders (Anderson, 1997), fig wasps (Whiting and Whiting, 2004) and stick insects (Whiting et al., 2003), digits in lizards (Brandley et al., 2008; Kohlsdorf and Wagner, 2006), developmental stages in salamanders (Chippindale et al., 2004; Mueller et al., 2004) and frogs (Wiens et al., 2007), teeth in lynx (Kurtén, 1963) and marsupial frogs (Wiens, 2011), thigh muscles in birds (Raikow, 1975), and oviparity in a clade of otherwise viviparous snakes (Lynch and Wagner, 2010), among many others.

Kurtén (1963) described one of the first examples of the reevolution of a lost complex character - the second lower molar $\left(\mathrm{M}_{2}\right)$ of the Eurasian lynx (Lynx lynx). Unlike all other extant felids, which lack $\mathrm{M}_{2}$, this molar is present in $3.4-27 \%$ of Eurasian lynx populations (Figure 1). Using an ad hoc parsimony argument, Kurtén suggested that $\mathrm{M}_{2}$ recently reevolved in the Eurasian lynx in association with the reevolution of the metaconid-talonid complex of the first 
RUNNING HEAD: LYNX M2 AND IRREVERSIBILE EVOLUTION

lower molar $\left(\mathrm{M}_{1}\right)$. This apparent break from Dollo's law was analyzed in a more phylogenetic framework by Werdelin (1987), who showed that $M_{2}$ was absent from other species of extant and extinct Lynx; following Kurtén, Werdelin proposed that the presence of $M_{2}$ was a true reversal. While the presence of $\mathrm{M}_{2}$ in Lynx lynx and its implications for Dollo's law were the subject of some debate (Kvam 1985), this example has become one of the most citied cases against strict enforcement of Dollo's law. However, Kurtén's proposal that $M_{2}$ reevolved in the Eurasian lynx has never been explicitly tested.

In this paper I reanalyze the evidence for the reevolution of the second lower molar $\left(\mathrm{M}_{2}\right)$ of the Eurasian lynx using explicit models of character evolution to evaluate Kurtén's proposal that Dollo's law was broken. First, I perform a molecular phylogenetic analysis of extant felids based on a published dataset of thirty nuclear and eight mitochondrial genes for 38 species of felids, including genera from each of the felid major lineages, plus seven feliform groups closely related to the Felidae to generate a phylogeny on which to test for revolution of $M_{2}$ in Lynx. Using this well-supported phylogeny, I reconstruct the phylogenetic history of the second molar in extant cats using parsimony- and likelihood-based models of character evolution and present strong statistical evidence that $M_{2}$ reevolved in the Eurasian lynx, reaffirming Kurtén's hypothesis. Finally I present a statistical model to explain the potential developmental genetic basis of reevolution, and simple developmental model to explain the mechanisms that may underlie the reappearance of $\mathrm{M}_{2}$ in the Eurasian lynx.

\section{Materials and Methods}

\section{Phylogenetic analysis}

We used the Johnson et al. (2006) dataset to generate a phylogeny for character reconstructions, which included 45 species and 23,920 nucleotide sites from thirty nuclear loci, APP, CALB, CHRNA, CLU, CMA, DGKG2, FES, GATA, GHR, GNAZ, GNB, HK1, NCL, PNOC, RAG2, RASA, SILV, TCP, TTR, ALAS, ATP7A, IL2RG, PLP, ZFX, SMCY, SRY3, SRY5, SRY, UBEY, ZFY, and eight mitochondrial loci 16s, ATP8, CYTB, ND1, ND2, ND4, ND5, and $t$-RNAs. Alignments were the same as in the original study, and regions of ambiguous alignment were excluded from further analysis. Bayesian phylogenetic analyses were performed with MR.BAYES v3.1 (Huelsenbeck and Ronquist, 2001) using a partitioning scheme that applied a separate GTR $+\Gamma$ substitution models to each gene and unlinking model parameters across each partition; more complex partitioning schemes, such as those dividing genes into codon positions, were 


\section{RUNNING HEAD: LYNX M2 AND IRREVERSIBILE EVOLUTION}

evaluated but generally were too computationally complex and failed to converge. Two independent Bayesian analyses were run for 10,000,000 generations each with four chains sampled every 1000 generations and a burnin of 2,500 trees. Run progress was visually checked with TRACER v1.4 by plotting the log-likelihoods of sampled generations, and the stability of parameter estimates (chain convergence) checked by ensuring that the standard deviation of split likelihood frequencies was below 0.01 while the potential scale reduction factor (PSRF) was close to 1.0 for all parameters.

\section{Character Evolution}

Parsimony and likelihood reconstructions of character evolution were performed with MESQUITE v2.01 (Maddison and Maddison, 2009). The rates of forward $\left(q_{01}\right)$ and reverse transitions $\left(q_{10}\right)$ under an asymmetrical likelihood model with separate rates for forward and reverse transitions estimated directly from the data (rMk2) and compared to an irreversible model (iMk2) that constrained the reverse rate to zero $\left(q_{10}=0\right)$. Because recent studies indicate that character associated changes in diversification rate can lead to erroneous rejection of irreversible models (Goldberg and lgić, 2008), we tested for character associated diversification using the BiSSE module (Maddison et al., 2007) implemented in MESQUITE v2.01. For both Markov and BiSSE models a stationary or "uninformative" prior was used to infer the state of the root node under reversible models while irreversible models fixed the state of the root node at 0 in the BiSSE analysis. The tree for character reconstructions was the consensus derived from the Bayesian analysis transformed into ultrametric tree with an arbitrary root age of 100 using the penalized likelihood method implemented in R8S v1.7 (Sanderson, 2003).

Parsimony based trait reconstructions were analyzed as unordered (reversible) and imposing the 'Dollo' model in MacClade. To compare the statistical support for different parsimony models we used the 'no common mechanism' model (NCM) of Tuffley and Steel (1997) to transform tree length into likelihoods. Calculation of the NCM model score followed equation 42 of Tuffley and Steel (1997),

$$
-\ln \mathrm{L}=\sum_{i=1}^{k}\left(l_{i}+1\right) \ln \left(r_{i}\right)
$$




\section{RUNNING HEAD: LYNX $M_{2}$ AND IRREVERSIBILE EVOLUTION}

where $l_{i}$ is the parsimony cost of the reconstruction given the tree, and $r_{i}$ is the number of states in character $k$; because only a single character is considered here, the NCM model is equivalent to a scaling of the parsimony tree length into -log likelihoods.

\section{Probabilistic models of escape from deleterious mutations}

Following Marshall, Raff, and Raff (1994), I calculated the probability that genes retain function during periods of "silencing". These authors proposed that the probability a gene (or regulatory element) retains a functional sequence in the absence of selection is

$$
P_{(\text {retain function })}=(f)^{\mu l t} \times(0.05)^{\eta l t}
$$

where $f$ is the probability that a nucleotide substitution is not inactivating, $\mu$ is the per nucleotide substitution rate in millions of years (Myr), $\eta$ is the rate of frameshifts (Myr), $I$ is the length of the sequence, $t$ is time (Myr) and 0.05 indicates that frameshifts in all but the last (most C-terminal) $5 \%$ of a gene are deleterious. Using empirical data, Marshall, Raff and Raff (1994) calculated a liberal $f$ to be 0.7 , and a moderate $\mu$ and $\eta$ to be 0.005 and 0.093 , respectively. Here we extend the Marshall, Raff, and Raff (1994) model from large protein coding genes to include sequences of size that are typical for both cis-regulatory elements and individual protein-protein interaction sites in proteins.

\section{Results and Discussion}

Unlike all other extant felids, 3.4-27\% of Eurasian lynx populations have a second lower molar ( $\mathrm{M}_{2}$; Figure 1). Using an ad hoc parsimony argument, Kurtén $(1953,1963)$ suggested that $\mathrm{M}_{2}$ recently reevolved in the Eurasian lynx, a proposition supported by Werdelin (1987) using a more phylogenetic framework. Here we reevaluated the evidence supporting the reevolution of $\mathrm{M}_{2}$ in the Eurasian lynx using a large, well-supported, phylogeny and contemporary models of character evolution.

\section{Phylogeny of the Felidae}

The Bayesian phylogeny inferred for the felids is very similar to those previously estimated using molecular data, and is nearly identical to the tree inferred by Johnson et al. (2006). We found that the majority nodes in the tree were supported by Bayesian posterior probabilities (BPP) of 1.0, with the exception being three nodes in the domestic cat lineage with BPP of 0.56, 0.71 , and 0.91 (Figure 2). Although the molecular dataset used to infer the phylogeny (Figure 
RUNNING HEAD: LYNX M2 AND IRREVERSIBILE EVOLUTION

2) is identical to the one by used Johnson et al. (2006), there are two differences between our tree topologies. We find that the Pallas cat is placed as the most basal lineage in the domestic cat clade, albeit with very poor support (BPP: 0.56), while Johnson et al. (2006) found the Pallas cat to be sister to the leopard lineage also with relatively poor support (BPP: 0.83). Second, we find that the puma lineage is placed as the sister clade to the lynxes (BPP: 1.0), rather than as sister to the leopard+domestic cat lineage as did Johnson et al. (2006), again with very poor support $(\mathrm{BPP}<0.50)$.

A preliminary analysis indicates that these different topologies result from different data partitioning schemes; Johnson et al. (2006) used as single $G T R+\Gamma+I$ model in their combined nuclear/mitochondrial Bayesian analysis while I used separate $G T R+\Gamma+I$ models for each codon position of protein coding genes and each stem and loop of RNA genes. Penalized likelihood analysis using our phylogeny and the 15 fossil calibrations used by Johnson et al. (2006) gives essentially the same divergence times as the original study and interested readers are referred to Johnson et al. (2006) for the systematic and biogeographic implications of felid phylogeny. We note, however, that these phylogenetic differences do not alter our inference of $M_{2}$ loss and gain in felids.

\section{Reevolution of $M_{2}$ in Eurasian lynx: Parsimony}

To infer whether explicit models of character evolution support the inference of $M_{2}$ reevolution, I reconstructed the evolutionary history of $M_{2}$ in cats using the well-supported molecular phylogeny plus the extinct Issoire lynx ( $L$. issiodorensis) of felids and parsimony reconstruction coding the Eurasian lynx as polymorphic for the presence of $M_{2}$. I found that a reversible model required only two steps (one basal loss in the stem-lineage of the Felidae, one regain in the Eurasian Lynx), while the irreversible 'Dollo' model, which disallows regains and thus imposes multiple losses of $\mathrm{M}_{2}$, required 10 steps (Table 1 and Figure 3). Comparison of NCM likelihoods between these two models indicates that there is considerably less support for the irreversible 'Dollo' model $(-\mathrm{LnL}=-7.62)$ than for the reversible model $(-\mathrm{LnL}=-2.08)$, indicating that there is essentially no support for the 'Dollo' model ( $\triangle A I C=11.09$; Table 1).

\section{Reevolution of $M_{2}$ in Eurasian lynx: Likelihood}

I also used two maximum likelihood models to test the irreversibility of $M_{2}$ loss: methods that compare an irreversible model to models that allow reversals without accounting for the 


\section{RUNNING HEAD: LYNX $M_{2}$ AND IRREVERSIBILE EVOLUTION}

effect of the character on rates of speciation and extinction (Mk2 models) and likelihood models that accommodate for the effect of a character on speciation and extinction rates (BiSSE models). All likelihood analysis were performed on the time calibrated tree, including only extant taxa.

I found that the irreversible Mk2 model (iMk2: $-L n L=-18.23$ ) was a significantly worse fit to the data than the reversible Mk2 model (rMk2: $-L n L=-9.41)$, indicating there was no support the irreversibility of $M_{2}$ loss (Table 1), i.e. a Dollo model $(\triangle A I C=18.18)$. In addition, the rMk2 model was only a marginally better fit to the data than a one-rate likelihood model (Mk1: -LnL = 10.14), suggesting that there was little gain in power by including two rates of character evolution $(\triangle \mathrm{AIC}=0.54)$. Reconstruction of the most recent common ancestor of the felids under both the rMk2 and Mk1 models indicated there was a high proportional likelihood it lacked $M_{2}$ (0.98), similarly reconstruction of the most recent common ancestor of the Lynx clade and Lynx lynx-Lynx pardinus lineages indicated there was a high likelihood these ancestors lacked $M_{2}$ (1.0 and 0.92, respectively; Figure 3).

Like parsimony, Mk models are prone to falsely reject irreversibility when a characters state influences diversification rates (Goldberg and Igić, 2008). To account for the possibility that $M_{2}$ presence/absence altered diversification rates in felids, confounding the inference of reversibility in parsimony and Mk models, we used the BiSSE model. The 'free' BiSSE model ($\mathrm{LnL}=-83.41$ ), which estimates different speciation and extinction rates for species with states 0 and 1 , was only 0.22 likelihood units better than a model constrained speciation rates to be shared between state 0 and $1(\triangle \mathrm{AIC}=0.96)$. Similarly, the 'free' model was only a slightly better fit to the data than models that constrained extinction rates $(\triangle \mathrm{AIC}=0.00)$ or both speciation and extinction rates $(\triangle \mathrm{AIC}=0.87)$ to be shared between state 0 and 1 . Thus, there is essentially no support for $\mathrm{M}_{2}$-dependent differences in diversification rates (Table 1). A BiSSE model that constrained $M_{2}$ loss to be irreversible (-LnL = -91.07), however, was a significantly worse fit to the data than the 'free' model $(\triangle \mathrm{AIC}=14.28$; Table 1).

\section{Is there a grace period on Dollo's law?}

Dollo's argument for irreversibility was not merely an empirical generalization from the facts of the history of life (phylogeny), rather for Dollo it was a "question of probabilities"; complex characters do not reevolve because this would require that the organism retrace, in 


\section{RUNNING HEAD: LYNX M2 AND IRREVERSIBILE EVOLUTION}

exactly the same order, an extremely large number of steps (Gould 1970). Thus, in the words of Muller, the loss of complex characters is irreversible because of "the sheer statistical improbability, amounting to an impossibility, of evolution ever arriving at the same complex genic end-result twice" (cited in Gould 1970). A developmental evolutionary basis for understanding Dollo's rational is that complex characters arise from the execution of complex developmental genetic regulatory networks; the absence of stabilizing selection will lead to mutational drift in the components of the network and their loss. Therefore, its unlikely that the genetic information for the development and function of the character can be maintained for long periods of time in the absence of the character (Bull and Charnov, 1985).

Using empirical data and a statistical model to assess the probability that silenced genes escape these kinds of deleterious mutations, Marshall, Raff and Raff (Marshall et al., 1994) estimated that reactivation of silenced genes was likely if the time of inactivation was on the order of 0.5-6 million years. In contrast, resurrecting long dormant genes (> 10 million years) was extremely unlikely because of the accumulation of multiple inactivating mutations unless gene function was maintained in other contexts. The survival of protein coding genes, however, may not be sufficient to explain the reemergence of dormant developmental programs because the development of complex characters also depends on the correct spatial and temporal expression of those genes and their interactions with other genes. For example, many multifunctional proteins have tissue specific cis-regulatory elements that direct their appropriate expression (Davidson, 2006) and specific protein-protein interaction motifs that mediate their biochemical functions (Neduva and Russell, 2005, 2006).

I quantified the probability that these smaller cis-regulatory element and protein-protein interaction motif sized regions escape deleterious mutations following the model of Marshall, Raff and Raff (1994). I found that 100bp regions, which approximate the size of individual regulatory elements, such as enhancers, and small protein interaction domains, have a half life of 25 million years, while 10bp regions, corresponding to the size of individual transcription factor binding-sites and protein-protein interaction sites in proteins such as transcription factors, have a half life of around 250 million years (Figure 4A). In stark contrast, short ( 1kb), intermediate $(\sim 3 \mathrm{~kb})$ and long $(\sim 5 \mathrm{~kb})$ protein coding genes that been silenced only have $50 \%$ chance of surviving lethal mutations after 2.5, 0.85 and 0.5 million years (Figure 4A), respectively. These data indicate that reactivation of silenced enhancers, individual transcription factor binding-sites, and protein-protein interaction motifs can occur after a much longer time 
RUNNING HEAD: LYNX M2 AND IRREVERSIBILE EVOLUTION

than an entire protein-coding gene.

\section{A simple developmental model of $M_{2}$ reevolution in Eurasian lynx}

The evolution and development of mammalian dentition has been intensely studied, leading to the generation of experimental- and developmental-based models that can predict patterns of mammalian tooth development. The development of mammalian molars, in particular, has been well-studied including the timing of molar initiation, mineralization, eruption, molar size, and number (Kavanagh et al., 2007). Mammalian molars, for example, develop sequentially in an anterior to posterior direction, resembling the development of serially homologous segmental structures. Kavanagh et al (2007) have described an activator-inhibitor model of sequential tooth development that describes the relative size and number of molars in mouse. In their model, an inhibitory cascade determines molar size differences along the jaw, such that the $\mathrm{M}_{2}$ always makes up one-third of total molar area, and that the first developing molar $\left(M_{1}\right)$ could inhibit the development of subsequent molars including $M_{2}$. Furthermore, the initiation of posterior molars depends on previous molars through a balance between intermolar inhibition and mesenchymal activation through BMP4 and Activin A (which are highly expressed in the mesenchyme at the onset of molar formation).

These data suggest that molar number was reduced from two to one in felids through an increase in the ability of $M_{1}$ to inhibit $M_{2}$ and/or a decrease in mesenchymal activation leading to the loss of $M_{2}$ (Figure $4 B$ ). Thus, the reevolution of $M_{2}$ in Eurasian lynx may be a relatively simple reversal of this developmental pattern such that there is a decrease in the ability of $M_{1}$ to inhibit $\mathrm{M}_{2}$ and/or an increase in mesenchymal activation leading to the emergence of $\mathrm{M}_{2}$ (Figure 4B). Remarkably Kurtén (1953) suggested that two processes, limits to the modification of existing features (i.e., evolutionary constraints) and linked development of phenotypic traits, may explain the reevolution of $\mathrm{M}_{2}$ in Eurasian lynx. Specifically, Kurtén (1953) suggested that selection for the enlargement of $M_{1}$ in Eurasian lynx, which is significantly larger in than other species, may be limited but reach a size that, through developmental linkage, raise $M_{2}$ above the threshold of phenotypic expression. Werdelin (1987) elaborated on this model to suggest that selection pressure for an enlarged molar region was greater in Eurasian lynx than enlargement of $M_{1}$ alone could accommodate, and $M_{2}$ reached the level of phenotypic expression through developmental linkage with $M_{1}$ in the molarization field (Butler 1939). Thus Kurtén and Werdelin proposed a extraordinarily prescient model for the reevolution of $M_{2}$ in 
RUNNING HEAD: LYNX M2 AND IRREVERSIBILE EVOLUTION

Eurasian lynx long before the establishment of mathematical and developmental models of mammalian dentition.

\section{Conclusions}

The long half-life of enhancers, transcription factor binding sites, and protein-protein interaction motifs suggests that evolutionary reversals are possible after much longer periods of loss than previously suspected. For example, the Cypriniform oral enhancer of $D / x 2 b$ has retained its ability to drive proper $D / x 2 b$ expression in oral teeth more than 50 millions after the loss of oral teeth in Cypriniforms indicating it has escaped from loss of function mutations (Jackman and Stock 2006). More remarkably, the rudiments of the developmental program leading to tooth formation are maintained in birds 70-80 million years after the loss of avian teeth (Harris et al., 2006). Thus, components of developmental programs can be maintained for extremely long periods of time in the absence of selection acting on an expressed structure, providing a starting point for the reemergence of lost characters. Similarly, shared developmental programs can maintain the developmental genetic information necessary for previously lost characters, such as $\mathrm{M}_{2}$ in Eurasian lynx, to reevolve even after long periods of absence (in this case 15 million years). 
RUNNING HEAD: LYNX $M_{2}$ AND IRREVERSIBILE EVOLUTION

\section{Acknowledgements}

VJL would like to thank G.P. Wagner (Yale University) for discussions on an earlier version of this manuscript.

\section{Author Contributions}

VJL conceived and designed the study, analyzed the data, drafted the manuscript and figures.

\section{References}

Anderson, N.M. (1997). Phylogenetic test of evolutionary scenarios: the evolution of flightlessness and wing polymorphism in insects. Mémoires du Muséum National d'Histoire Naturelle 173, 91-108.

Brandley, M.C., Huelsenbeck, J.P., and Wiens, J.J. (2008). Rates and patterns in the evolution of snake-like body form in squamate reptiles: evidence for repeated re-evolution of lost digits and long-term persistence of intermediate body forms. Evolution 62, 2042-2064.

Bull, J.J., and Charnov, L. (1985). On irreversible evolution. Evolution 39, 1149-1155.

Butler, P. M. (1939) Studies of the mammalian dentition. Differentiation of the post-canine dentition. Proc. Zool. Soc., 109B, 1-36

Chippindale, P.T., Bonett, R.M., Baldwin, A.S., and Wiens, J.J. (2004). Phylogenetic evidence for a major reversal of life-history evolution in Plethodontid salamanders. Evolution 58, 28092822.

Coates, M., and Rutta, M. (2000). Nice snake, shame about the legs. Trends in Ecology \& Evolution 15, 503-507.

Davidson, E.H. (2006). The Regulatory Genome: Gene Regulatory Networks In Development And Evolution 1st edn (Burlington, MA: Academic Press).

Desutter-Grandcolas, L. (1993). The cricket fauna of Chiapanecan caves (Mexico): systematics, 
RUNNING HEAD: LYNX $M_{2}$ AND IRREVERSIBILE EVOLUTION

phylogeny and the evolution of troglobitic life (Orthoptera, Grylloidae, Phalangopsidae, Luzarinae). International Journal of Speleology 22, 1-82.

Dingle, R.V. (2003). Some paleontological implications of putative, long tern, gene reactivation. Journal of the Geological Society, London, 160, 1-4.

Goldberg, E.E., and Igić, B. (2008). On phylogenetic tests of irreversbile evolution. Evolution 62, $11,2727-41$.

Harris, M.P., Hasso, S.M., Fergurson, M.W.J., Fallon, J.F. (2006). The development of archosaurian first-generation teeth in a chicken mutant. Current Biology 4, 21, 371-377.

Huelsenbeck, J.P., and Ronquist, F. (2001). MRBAYES: Bayesian inference of phylogenetic trees. Bioinformatics 17, 754-755.

Hunt, G. (2007). Morphology, ontogeny, and phylogenetics of the genus Poseidonamicus (Ostracoda : Thaerocytherinae). Integrative and Comparative Biology 43, 522-530.

Jackman W. R,. Stock, D.W. (2006). Transgenic analysis of Dlx regulation in fish tooth development reveals evolutionary retention of enhancer function despite organ loss. PNAS 103, 51, 19390-19395.

Johnson, W.E., et al. (2006). The late miocene radiation of modern felidae: a genetic assessment. Science 311, 5757, 73-7.

Kavanagh K.D., Evans, A.R., Jernvall, J. (2007). Predicting evolutionary patterns of mammalian teeth from development. Nature 499, 27, 427-432.

Kohlsdorf, T., and Wagner, G.P. (2006). Evidence for the reversibility of digit loss: a phylogenetic study of limb evolution in Bachia (Gymnophthalmidae: Squamata). Evolution 60, 1896-1912.

Kurtén, B. (1953). On the variation and population dynamics of fossil and recent mammal populations. Acta Zool. Fenn, 76, 1-122.

Kurtén, B. (1963). Return of a lost structure in the evolution of the felid dentition. Soc Sci Fenn Comm Biol 4, 1-12. 
RUNNING HEAD: LYNX $M_{2}$ AND IRREVERSIBILE EVOLUTION

Laurent, R.F. (1983). Irreversibility: A Comment on Macbeth's Interpretations. Systematic Zoology 32, 75.

Lynch, V.J., and Wagner, G.P. (2010). Did egg-laying boas break dollo's law? Phylogenetic evidence for reversal to oviparity in sand boas (eryx: boidae). Evolution 64, 207-216.

Maddison, W.P., and Maddison, D.R. (2009). Mesquite: a modular system for evolutionary analysis.

Maddison, W.P., Midford, P.E., and Otto, S.P. (2007). Estimating a binay character's effect on speciation and extinction. Systematic Biology 56, 701-710.

Marshall, C.R., Raff, E.C., and Raff, R.A. (1994). Dollo's law and the death and resurrection of genes. Proceedings of the National Academy of Sciences, USA 91, 12283-12287.

Werdelin, L. (1987). Supernumerary teeth in Lynx lynx and the irreversibility of evolution. J. Zool., Lond. 211, 259-266.

Mueller, R.L., Macey, R.J., Jaekel, M., Wake, D.B., and Boore, J.L. (2004). Morphological homoplasy, life history evolution, and historical biogeography of plethodontid salamanders inferred from complete mitochondrial genomes. Proceedings of the National Academy of Sciences, USA 101, 13820-13825.

Neduva, V., and Russell, R.B. (2005). Linear motifs: Evolutionary interaction switches. FEBS Letters 579, 3342-3345.

Neduva, V., and Russell, R.B. (2006). Peptides mediating interaction networks: new leads at last. Current Opinion in Biotechnology 17, 465-471.

Oakley, T.H. (2003). On homology of arthropod compound eyes. . Integrative and Comparative Biology 43, 522-530.

Raikow, S.J. (1975). The evolutionary reappearance of ancestral muscles as developmental anomalies in two species of birds. Condor 77, 514-517.

Sanderson, M.J. (2003). r8s: inferring absolute rates of molecular evolution and divergence times in the absence of a molecular clock. Bioinformatics 19, 301-302. 
RUNNING HEAD: LYNX $M_{2}$ AND IRREVERSIBILE EVOLUTION

West-Ebehard, M. (2003). Developmental plasticity and evolution. (Oxford, U.K.: Oxford University Press).

Whiting, M.F., Bradler, S., and Maxwell, T. (2003). Loss and recovery of wings in stick insects. Nature 421, 264-267.

Whiting, M.F., and Whiting, A.S. (2004). Is wing recurrence really impossible?: a reply to Trueman et al. Systematic Entomology 29, 140-141.

Wiens, J.J. (2011). re-evolution of lost mandibular teeth in frogs after more than 200 million years, and re-evaluating dollo's law. Evolution 65, 1283-1296.

Wiens, J.J., Kuczynski, C., Duellman, W.E., and Reeder, T.W. (2007). Loss and re-evolution of complex life cycles in marsupial frogs: does ancestral trait reconstruction mislead? Evolution 61, 1886-1899. 
RUNNING HEAD: LYNX M2 AND IRREVERSIBILE EVOLUTION

Table 1. Model comparisons testing the irreversibility of molar loss. Speciation $(\lambda)$, extinction $(\boldsymbol{\mu})$ and character transition $(\boldsymbol{q})$ rate estimates are reported based on the time calibrated tree with an arbritary root age of 100 . The likelihood of each model $(\ln L)$ is shown, followed by the $\triangle \mathrm{AICs}$; the best models are in bold. Note that the BiSSE model does not support trait dependent diversification rates, therefore our conclusions on reversibility are based on the Mk2 model. BiSSE and parsimony results are shown for comparison only. The No Common Mechanism (NCM) score is a scaling of the parsimony tree length into -log likelihoods and is shown for comparison to the models. Note that the parsimony model includes the extinct Issoire lynx ( $L$. issiodorensis) that is not included in either the BiSSE or Mk models.

\begin{tabular}{|c|c|c|c|c|c|c|c|c|}
\hline Model & $\lambda_{0}$ & $\lambda_{1}$ & $\mu_{0}$ & $\mu_{1}$ & $q_{01}$ & $q_{10}$ & $-\ln L$ & $\Delta \mathrm{AIC}$ \\
\hline \multicolumn{9}{|l|}{ BiSSE } \\
\hline Free & 0.583 & 0.476 & $1.3 \times 10^{-4}$ & $2.3 \times 10^{-5}$ & 0.046 & 0.015 & -83.41 & 0.00 \\
\hline Single $\lambda$ & 0.492 & & $1.3 \times 10^{-6}$ & 0.008 & 0.040 & 0.022 & -83.93 & 0.96 \\
\hline Single $\mu$ & 0.587 & 0.475 & $1.2 \times 10^{-6}$ & & 0.047 & 0.015 & -83.41 & 0.00 \\
\hline Single $\lambda \& \mu$ & 0.489 & & $3.5 \times 10^{-6}$ & & 0.036 & 0.023 & -83.88 & 0.87 \\
\hline Irreversible & 0.565 & 0.449 & $1.0 \times 10^{-4}$ & $3.2 \times 10^{-6}$ & 0.365 & 0.000 & -91.07 & 14.28 \\
\hline \multicolumn{9}{|l|}{ Mk1 } \\
\hline One Rate & & & & & 0.024 & & -10.14 & 0.00 \\
\hline \multicolumn{9}{|l|}{ Mk2* } \\
\hline Reversible & - & - & - & - & 0.014 & 0.087 & -9.41 & 0.54 \\
\hline Irreversible & - & - & - & - & 0.361 & 0.000 & -18.23 & 18.18 \\
\hline Parsimony & \multicolumn{2}{|c|}{ Cost Gain } & \multicolumn{2}{|c|}{ Cost Reversal } & \multicolumn{2}{|c|}{ Tree Length } & $N C M$ & \\
\hline Reversible & \multicolumn{2}{|c|}{1} & \multicolumn{2}{|c|}{1} & \multicolumn{2}{|c|}{2} & -2.08 & 0.00 \\
\hline Dollo P (extant) & \multicolumn{2}{|c|}{1} & \multicolumn{2}{|c|}{$\infty$} & \multicolumn{2}{|c|}{10} & -7.62 & 11.09 \\
\hline Dollo P (fossil) & \multicolumn{2}{|c|}{1} & \multicolumn{2}{|c|}{$\infty$} & \multicolumn{2}{|c|}{13} & -9.70 & 15.25 \\
\hline Polymorphism P & \multicolumn{2}{|c|}{1} & \multicolumn{2}{|c|}{1} & \multicolumn{2}{|c|}{13} & -15.38 & 26.60 \\
\hline
\end{tabular}




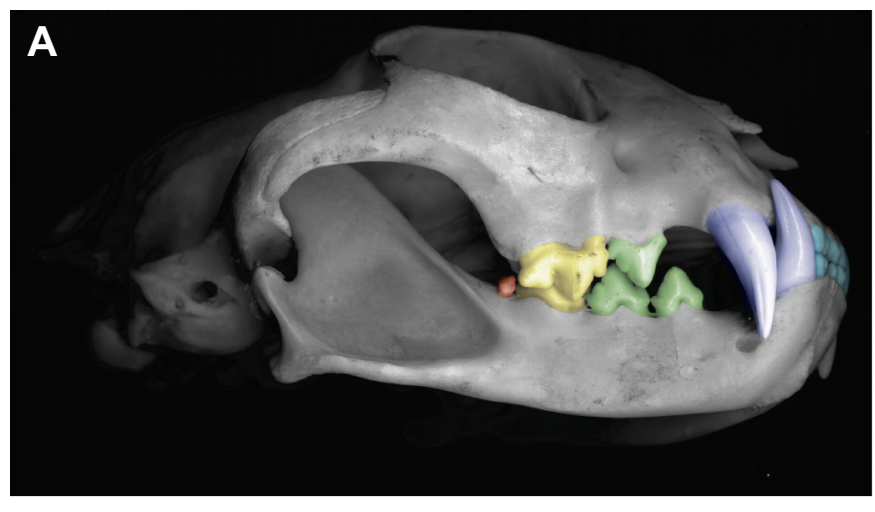

\section{B}
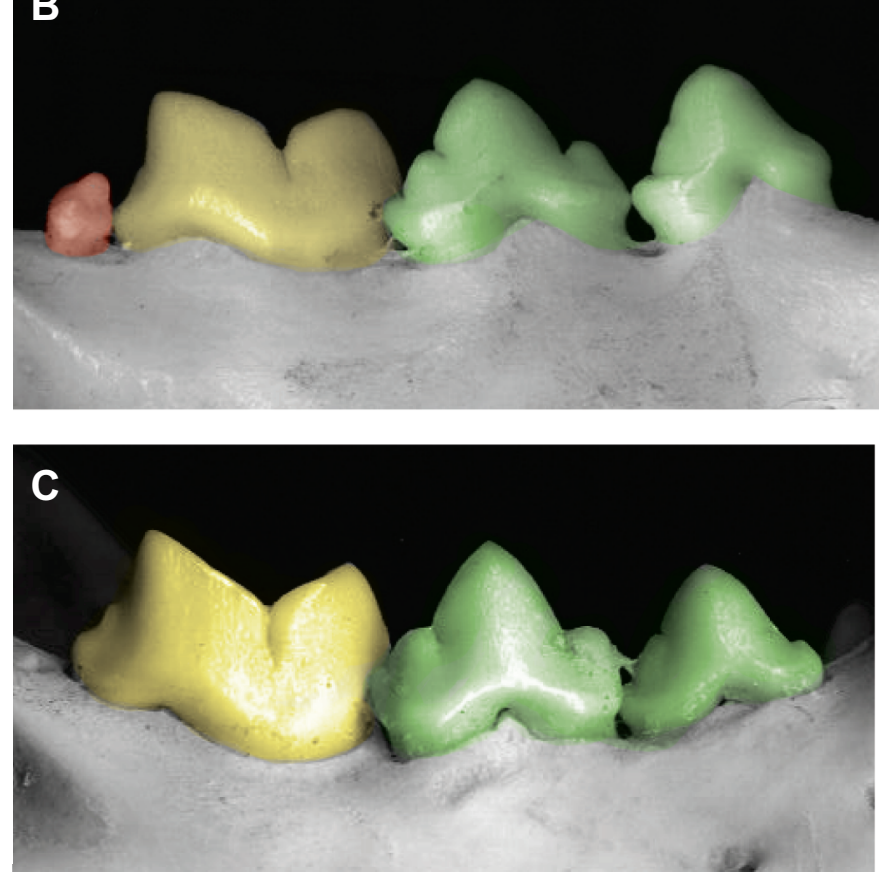

Figure 1. Dental variation in the Eurasian lynx (Lynx lynx). (A) Articulated skull, with incisors colored light blue, canines dark blue, premolars green, the first molar $\left(\mathrm{M}_{1}\right)$ yellow, and the second molar $\left(\mathrm{M}_{2}\right)$ orange. (B) Close up of a mandible with $\mathrm{M}_{2}$ colored orange. (C) Close up of a mandible without $\mathrm{M}_{2}$ colored. 
bioRxiv preprint doi: https://doi.org/10.1101/2021.02.04.429820; this version posted February 5, 2021. The copyright holder for this preprint (which was not certified by peer review) is the author/funder. All rights reserved. No reuse allowed without permission.

RUNNING HEAD: LYNX $M_{2}$ AND IRREVERSIBILE EVOLUTION

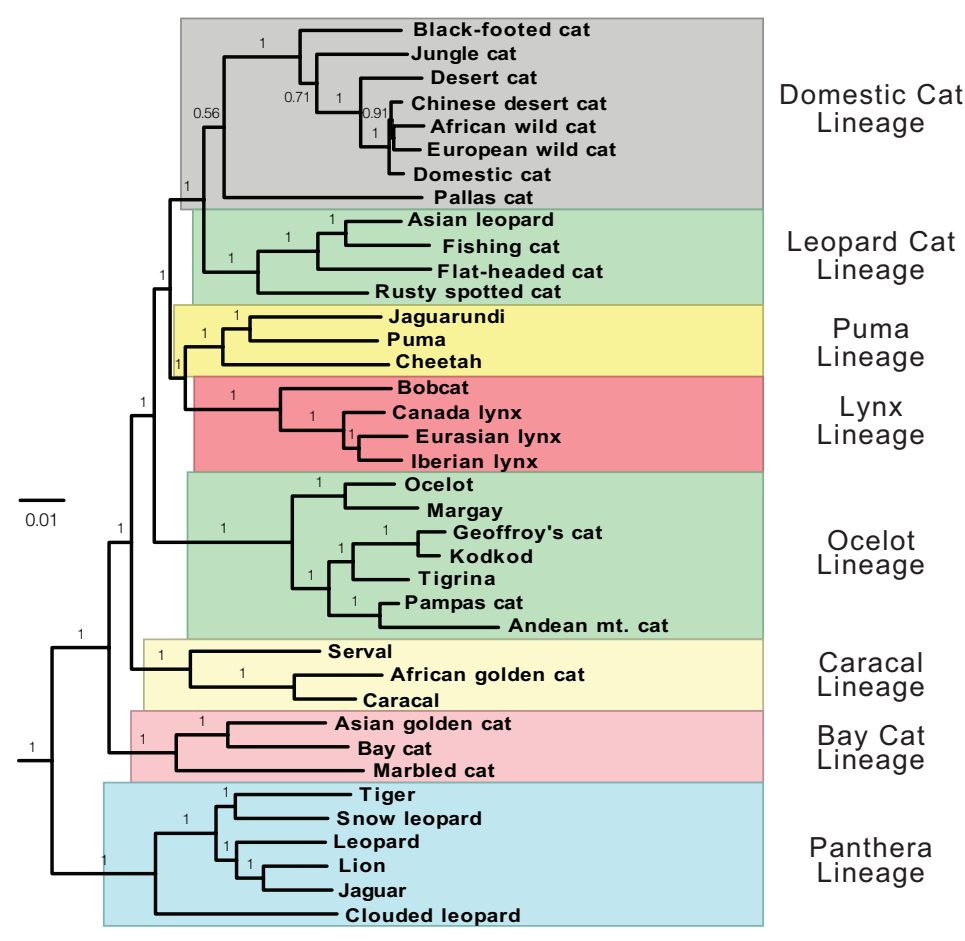

Figure 2. Felid phylogeny. Bayesian phylogeny of extant cats based on a concatenated alignment including 45 species and 23,920 nucleotide sites from 30 nuclear loci and eight mitochondrial loci. Branch lengths are proportional to substitutions per site, Bayesian Posterior Probabilities (BPP) for each branch. Major lineages are named and highlighted.

Bay Cat

Panthera Lineage 


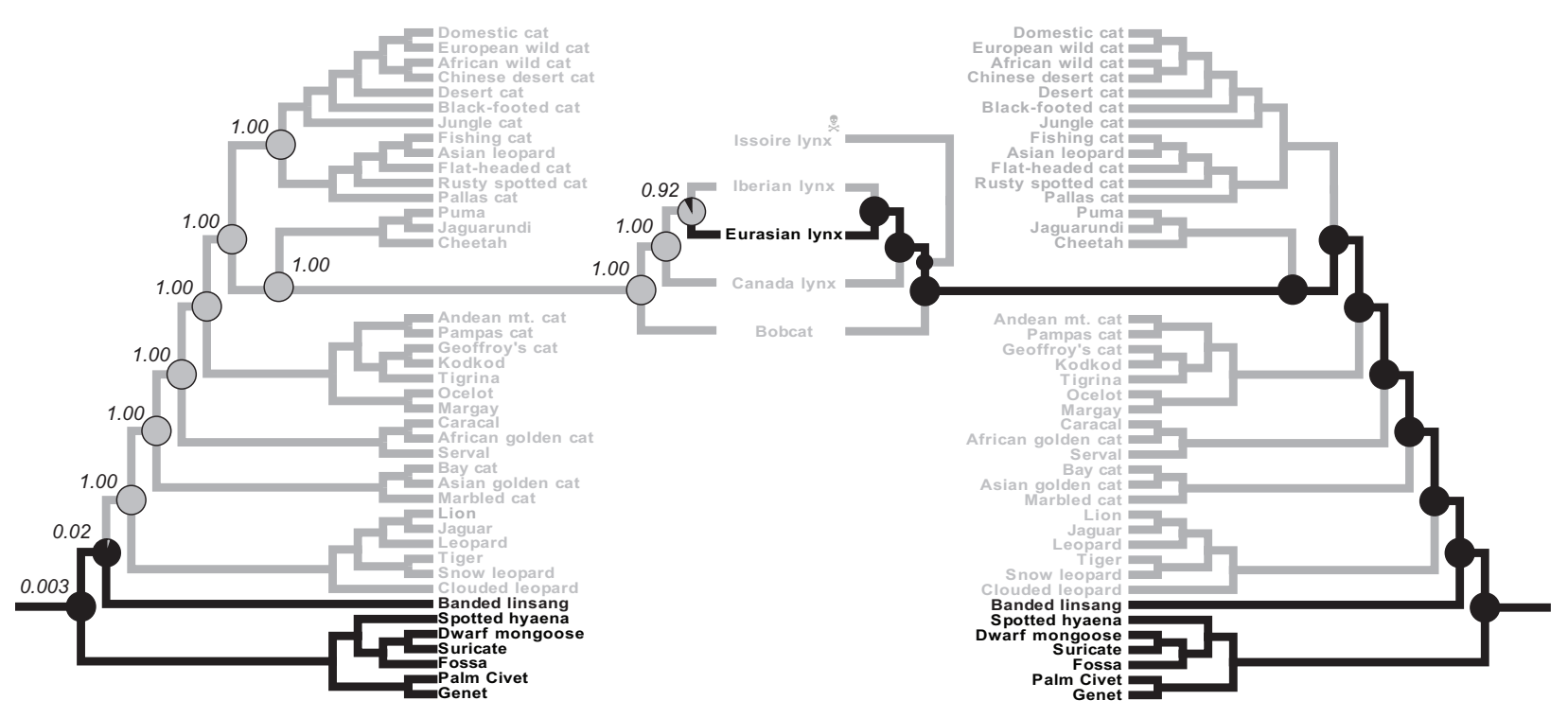

Figure 3. Phylogenetic support for the reevolution of $\mathbf{M}_{2}$ in Eurasian lynx. Left, Reconstruction of ancestral states under the reversible Mk2 model. The probability of $M_{2}$ presence (black) and absence (grey) is shown at each ancestral node as a pie chart colored by the probability of each state. The probability of $\mathrm{M}_{2}$ absence is shown at each node. Branches are colored according to the reversible parsimony model. Right, Reconstruction of ancestral states under the irreversible Mk2 model. The probability of $M_{2}$ presence (black) and absence (grey) is shown at each ancestral node as a pie chart colored by the probability of each state. Branches are colored according to ancestral states under an irreversible (Dollo) parsimony model. 


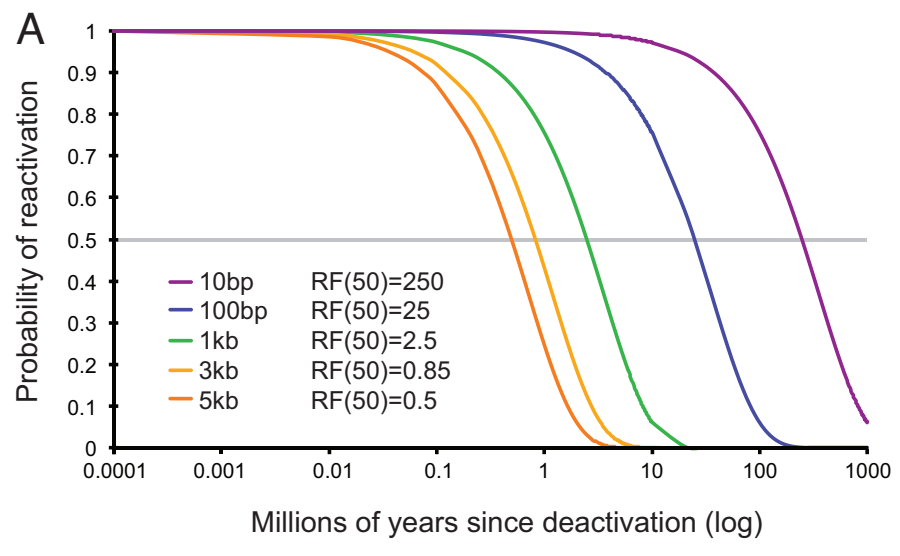

B

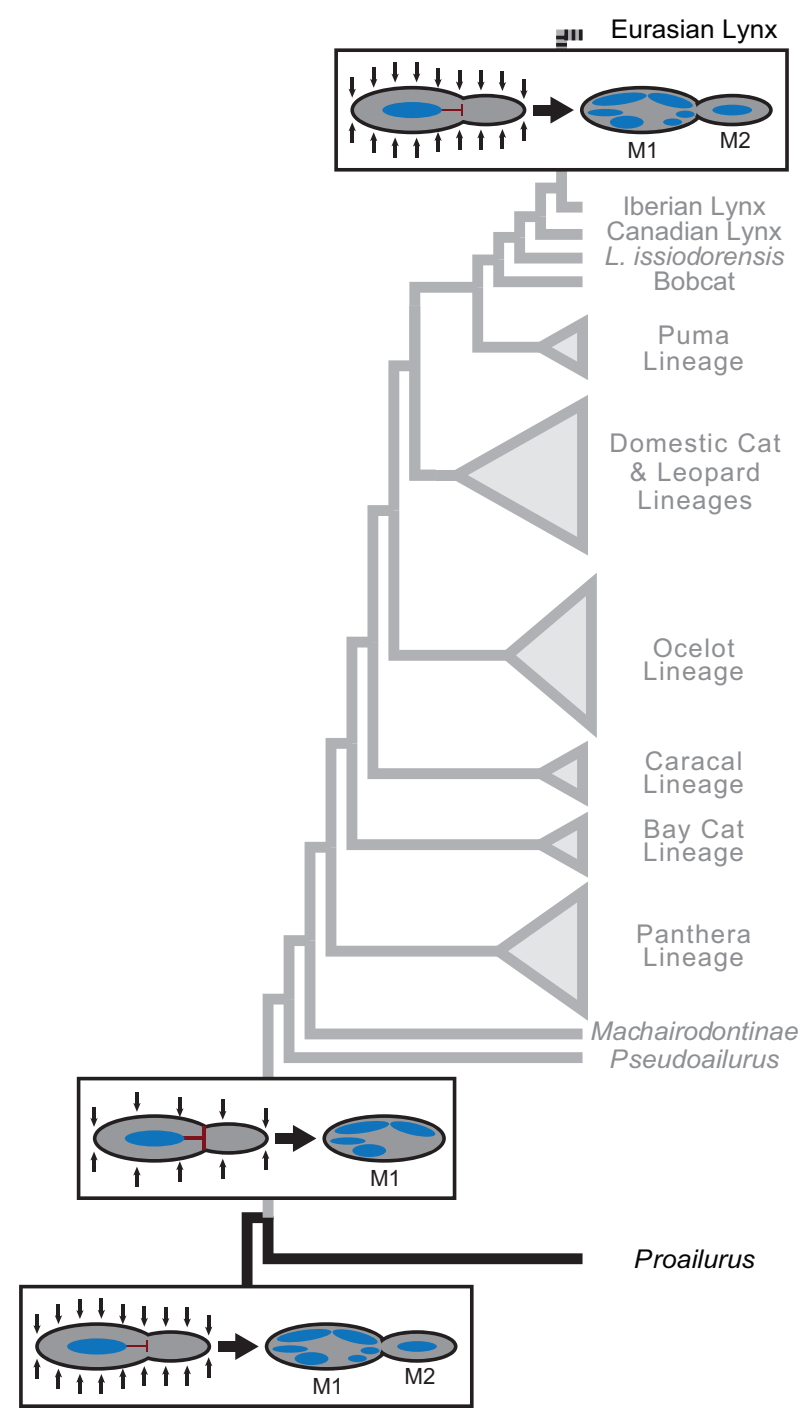

Figure 4. A simple model for the reevolution of lost complex traits. (A) The probability that functional DNA units, such as genes, regulatory elements, transcription factor binding sites, and regions encoding protein-protein interactions within genes, can be reactivated after they are deactivated. Note that smaller elements have a greater probability of reactivation. (B) $A$ developmental model for the loss of $M_{2}$ in cats and reevolution of $\mathrm{M}_{2}$ in Eurasian lynx. Molar number is regulated by an activatorinhibitor model in the molarization field (black box), in which an inhibitory signal from the first developing molar $\left(M_{1}\right)$ can inhibit (red blunt arrow) the development of $M_{2}$ and mesenchymal activation signals (black arrows) can promote the formation of molars distal to $M_{1}$. Molar number can be reduced from two to one in felids (gray lineages) through an increase in the ability of $M_{1}$ to inhibit (large red blunt arrow) $M_{2}$ and/or a decrease in mesenchymal activation (fewer black arrows) leading to the loss of $M_{2}$. Reevolution of $M_{2}$ in Eurasian lynx may have occurred by a reversal of this developmental pattern such that there is a decrease in the ability of $M_{1}$ (small red blunt arrow) to inhibit $\mathrm{M}_{2}$ and/or an increase in mesenchymal activation (more black arrows) leading to the emergence of $\mathrm{M}_{2}$. 